\title{
FATORES DE RISCO PARA QUEDAS EM IDOSOS HOSPITALIZADOS
}

\author{
J.V.B. CABRAL ${ }^{1 *}$, C.M.S. SILVA ${ }^{2}$, D.J.S. BISPO ${ }^{2}$ e E.M. SILVA ${ }^{1}$ \\ 1Universidade Federal de Pernambuco - UFPE \\ 2Centro Universitário Maurício de Nassau - UNINASSAU - Pernambuco \\ jvbcabral@gmail.com*
}

Artigo submetido em janeiro/2015 e aceito em abril/2016

DOI: $10.15628 /$ holos.2016.2668

\section{RESUMO}

O processo de envelhecer e a população idosa apresentam-se numa crescente, sendo necessário a avaliação e acompanhamento constante desta população e os fatores que influenciam na sua saúde, ocorrência de doenças e fatores adversos. Este estudo objetivou descrever quais os fatores de risco para a queda mais frequentes nos idosos hospitalizados. Para tanto, o estudo fundamentou-se metodologicamente em Mendes, Silveira e Galvão (2008) e Souza, Silva e Carvalho (2010). Tratando-se de uma revisão integrativa, com coleta de dados realizada a partir de fontes secundárias, por meio de levantamento e análise bibliográfica de publicações realizadas entre os anos de 2010 a 2014. Para o levantamento dos artigos foi realizada busca nas bases de dados da Biblioteca Virtual de Saúde (BVS), Literatura Latino-Americana e do Caribe em Ciências da Saúde
(LILACS) e Biblioteca Científica Eletrônica On-line (SciELO). Neste sentido, os achados deste estudo apontaram que apesar dos fatores de riscos serem reconhecidos, há ainda lacunas em relação aos estudos sobre a referida temática, pois apenas nove dos trinta trabalhos analisados tratam desta discussão efetivamente, o que comprova, não apenas a necessidade de mais estudos, como uma maior ampliação das ações desenvolvidas para saúde dos idosos. Conclui-se que as quedas em idosos hospitalizados ocorrem devido à falta de medidas preventivas que podem ser implementadas pelas instituições, assim como, os órgãos competentes devem intensificar os programas voltados para a população idosa em geral.

PALAVRAS-CHAVE: Síncope, Risco, Idoso, Hospitalização.

\section{RISK FACTORS FOR FALLS IN ELDERLY HOSPITALIZED}

\begin{abstract}
The process of aging and the elderly population presents a growing, requiring constant monitoring and evaluation of this population and the factors that influence their health, occurrence of diseases and adverse factors. This study aimed to describe what the risk factors for the most common drop in hospitalized elderly. Therefore, the study was based on methodologically Mendes Silveira and Galvão (2008) and Souza, Silva and Carvalho (2010). In the case of an integrative review, with data collection performed from secondary sources, through survey and literature review of publications carried out between the years 2010 to 2014. For the survey of the articles was conducted a search in databases Virtual Health library
\end{abstract}

(VHL), Latin American and Caribbean Health Sciences (LILACS) and Scientific Electronic library online (SciELO). In this sense, the findings of this study show that despite the risk factors are recognized, there are still gaps in relation to studies on this theme, as only nine of the thirty works analyzed treat this discussion effectively, which proves not only the need for more studies, such as greater expansion of the actions developed for health of the elderly. We conclude that the falls in hospitalized elderly occur due to lack of preventive measures that can be implemented by the institutions, as well as the competent bodies should intensify programs for the elderly population in general.

KEYWORDS: Syncope, Risk, Elderly, Hospitalization. 


\section{INTRODUÇÃO}

Segundo dados da Organização Mundial de Saúde (OMS), o envelhecimento populacional caracteriza-se pelo aumento da proporção de idosos em relação à população total, sendo considerado um fenômeno mundial, e o que era no passado um privilégio de poucos, agora passou a ser uma experiência vivida por um número cada vez mais crescente de pessoas ${ }^{1}$.

No Brasil, existem aproximadamente 23,7 milhões de pessoas com idade igual ou superior a 60 anos, o que representa pelo menos $12,6 \%$ da população brasileira. A população está envelhecendo, e de acordo com os levantamentos do Instituto Brasileiro de Geografia e Estatística (IBGE), estima-se que em 2050 existam aproximadamente 2 bilhões de pessoas com 60 anos ou mais no mundo e que grande parte resida em países em desenvolvimento ${ }^{2}$.

Durante o envelhecimento, a capacidade de funcionamento do organismo diminui de modo natural e fisiológico. Trata-se de um evento primário comum a todos os indivíduos da mesma espécie, passível de interações entre os genes e o ambiente. Assim, diversos agentes podem adiantar ou atrasar as modificações orgânicas envolvendo a interação de determinantes internos, como o patrimônio genético e dos determinantes externos, como o estilo de vida, a educação e o ambiente em que o indivíduo vive ${ }^{3}$.

O processo do envelhecer promove mudanças no organismo que levam à diminuição da acuidade visual e auditiva, da estatura e massa muscular, na velocidade dos reflexos; retardamento e/ou dificuldade no aprendizado e na memória e no uso de inúmeras medicações. É válido destacar a ocorrência das Doenças Crônicas Não Transmissíveis (DCNT), como característica marcante da população idosa, estas que muitas vezes deixam sequelas, que prejudicam ou impossibilitam a realização das atividades diárias, levando o idoso à dependência de terceiros e frequentes hospitalizações ${ }^{4}$.

Quando a hospitalização é necessária, o idoso tende a perder seu referencial principal, sua casa e a presença integral a sua família, de modo que a mudança ambiental torna-se outra dificuldade $\mathrm{e}^{5}$. As diferenças entre o hospital e a sua residência, com alterações no ambiente e disposição de objetos, representa uma mudança importante, principalmente para os mais senis ou com maior dificuldade de adaptação. Nesse contexto, as alterações das condições físicas, ligadas ou não à patologia que levou ao internamento estão à mercê de situações de maior fragilidade, com comprometimento físico e psicológico ${ }^{6}$.

O papel da família é importante em qualquer estágio da vida, mas é fundamental no momento da hospitalização, pois nesta situação as relações afetivas de esperança, segurança e o apoio são necessárias ${ }^{3}$. As instituições hospitalares, por sua vez, têm como dever proporcionar um ambiente seguro e acolhedor aos idosos funcionalmente dependentes ou não e com isto assegurar a manutenção da autonomia, conforto, prevenção de doenças, inclusão social e principalmente uma assistência à saúde eficiente e de qualidade ${ }^{7}$.

Diante dos agravos a saúde e com base nas informações anteriores, é importante enfatizar o fator queda, evento adverso comumente presente nos ambientes hospitalares que pode ser definida como deslocamento não intencional do corpo para um nível inferior à posição inicial, sem 
correção do tempo hábil ${ }^{8}$. Neste sentido, entendemos que qualquer pessoa apresenta risco de sofrer queda, no entanto, sabe-se que para os idosos o percentual desse risco aumenta, levandoos à incapacidade, deformidades e até a morte.

A queda no ambiente hospitalar é motivo de intensas discussões na tentativa de adotar estratégias para contê-las e desenhar um perfil de pacientes que sofrem esse evento ${ }^{7}$. Tal preocupação permite a instituição hospitalar o desenvolvimento de práticas mais seguras e geração de indicadores importantes. Todavia, tal fato ainda apresenta-se como um desafio para as instituições, que necessitam assumir atitudes eficazes no âmbito da investigação, formação e implementação de medidas preventivas ${ }^{9}$.

Com base nesta problemática, onde o processo de envelhecer e a população idosa apresentam-se numa crescente, é necessária a avaliação e acompanhamento constante desta população e os fatores que influenciam na sua saúde, ocorrência de doenças e fatores adversos. Este estudo objetivou descrever quais os fatores de risco mais frequentes para queda nos idosos hospitalizados.

\section{METODOLOGIA}

Este estudo fundamentou-se na revisão integrativa descritivo-exploratória, que consiste na elaboração de análise literária e na promoção de discussões sobre resultados de pesquisas. 0 estudo de revisão integrativa é realizado através de seis etapas: seleção de questões para a revisão; seleção dos estudos que irão constituir a amostra da revisão; definição das características primárias que compõem a amostra da revisão; análise dos achados dos artigos; interpretação dos resultados; e relato da revisão, proporcionando exame crítico dos achados de acordo com Mendes, Silveira e Galvão, 2008; SOUZA, SILVA e Carvalho, 2011. Nesta revisão, optou-se por seguir essas etapas.

A coleta de dados foi realizada a partir de fontes secundárias, por meio de levantamento e análise bibliográfica de publicações realizadas entre os anos de 2010 a 2014 . Período escolhido com intuito de obter publicações recentes com discussões atualizadas a respeito da temática. Para realização da busca dos artigos, o delineamento deste estudo guiou-se pela seguinte pergunta condutora: "Quais os fatores de risco para queda em idosos hospitalizados?" e através de busca pelas palavras-chave na Biblioteca Virtual em Saúde - BVS por meio das fontes de dados Centro Latino-Americano e do Caribe de Informação em Ciências da Saúde - LILACS e associada ao repositório Scientific Electronic Library Online - SciELO.

Consultando-se os Descritores em Ciências da Saúde (DeCS/MeSH), foram selecionadas as palavras-chave "Síncope, Risco, Idoso, Hospitalização" combinando-os de forma conjunta com a utilização do operador booleano "and".

Foram encontrados 30 artigos, destes 09 foram selecionados como amostra final e discussão e os demais como fomento para discussão dos achados. Os critérios de inclusão definidos para a seleção foram: artigos publicados em língua portuguesa, disponíveis on-line de forma gratuita, com texto completo, que retratassem a temática abordada, artigos publicados e indexados nos referidos bancos de dados no período já mencionado. Excluíram-se artigos de opinião e editoriais ou não conformes com os critérios de inclusão. 
A coleta dos dados guiou-se pelos critérios acima estabelecidos e pela leitura crítica dos resumos de cada artigo e posterior leitura na íntegra, caso o mesmo fosse coeso com a temática proposta. Para a expressão dos dados dos artigos, foi elaborado instrumento (Quadro 1) que contém os seguintes itens: ano, base/revista, título, temática e evidências.

\section{RESULTADOS E DISCUSSÃO}

A amostra final desta revisão foi constituída por nove artigos científicos, selecionados pelos critérios de inclusão e exclusão previamente estabelecidos. Destes, seis foram encontrados na base de dados SciELO e três na base LILACS. Das publicações selecionadas, observou-se que cinco foram publicadas em periódicos que contemplam a área da enfermagem, dois em periódicos da saúde pública, um na grande área de ciências da saúde e um na área de gerontologia e geriatria.

Quadro 1: Artigos levantados nas bases de dados da BVS - LILACS e SciELO sobre os fatores de risco para queda em idosos hospitalizados.

\begin{tabular}{|c|c|c|c|c|c|}
\hline Ano & $\begin{array}{l}\text { Base / } \\
\text { Revista }\end{array}$ & Autor & Título & Temática & Evidências \\
\hline 2010 & $\begin{array}{l}\text { LILACS / } \\
\text { Revista } \\
\text { Baiana de } \\
\text { Saúde } \\
\text { Pública }\end{array}$ & $\begin{array}{l}\text { Queiroz, L; } \\
\text { Lira,S; } \\
\text { Sasaki,A. }\end{array}$ & $\begin{array}{l}\text { Identificação do risco } \\
\text { de quedas pela } \\
\text { avaliação da } \\
\text { mobilidade funcional } \\
\text { em idosos } \\
\text { hospitalizado } \\
\end{array}$ & $\begin{array}{l}\text { Foram estimados os } \\
\text { riscos de queda em } \\
\text { idosos hospitalizados } \\
\text { em Salvador-BA. }\end{array}$ & $\begin{array}{l}\text { A idade avançada e o uso } \\
\text { de auxiliar de marcha } \\
\text { mostraram-se } \\
\text { como fatores associados } \\
\text { ao aumento da propensão } \\
\text { à queda. }\end{array}$ \\
\hline 2010 & $\begin{array}{c}\text { SciELO / } \\
\text { Revista de } \\
\text { Enfermagem } \\
\text { Referência }\end{array}$ & $\begin{array}{l}\text { Almeida, } \\
\text { R.A.R;Abreu,C. } \\
\text { F.C;Mendes,A. } \\
\text { M.O.C. }\end{array}$ & $\begin{array}{l}\text { Quedas em doentes } \\
\text { hospitalizados: } \\
\text { Contributos para } \\
\text { uma prática baseada } \\
\text { na prevenção }\end{array}$ & $\begin{array}{l}\text { As quedas como o } \\
\text { acidente que elevam } \\
\text { o aumento do tempo } \\
\text { de internamento. }\end{array}$ & $\begin{array}{c}\text { A queda como evento } \\
\text { multifatorial de causas } \\
\text { individuais ou ambientais. }\end{array}$ \\
\hline 2011 & $\begin{array}{c}\text { SciELO / } \\
\text { Revista } \\
\text { Gaúcha } \\
\text { Enfermagem }\end{array}$ & $\begin{array}{c}\text { Costa,S.G.R.F; } \\
\text { et al }\end{array}$ & $\begin{array}{l}\text { Caracterização das } \\
\text { quedas do leito } \\
\text { sofridas por } \\
\text { pacientes internados } \\
\text { em um hospital } \\
\text { universitário. }\end{array}$ & $\begin{array}{l}\text { Caracterizou o perfil, } \\
\text { fatores de risco e } \\
\text { diagnósticos de } \\
\text { enfermagem de } \\
\text { pacientes que } \\
\text { sofreram quedas do } \\
\text { leito durante a } \\
\text { internação. }\end{array}$ & $\begin{array}{l}\text { Os achados assemelham- } \\
\text { se ao descrito na literatura } \\
\text { mundial, reforçando a } \\
\text { necessidade de instalação } \\
\text { de medidas preventivas } \\
\text { para quedas e mitigação } \\
\text { dos riscos. }\end{array}$ \\
\hline 2011 & $\begin{array}{l}\text { SciELO / } \\
\text { Geriatria\& } \\
\text { Gerontologi } \\
\text { a }\end{array}$ & $\begin{array}{l}\text { Sales, M.V. C; } \\
\text { et al }\end{array}$ & $\begin{array}{l}\text { Efeitos adversos da } \\
\text { internação hospitalar } \\
\text { para o idoso. }\end{array}$ & $\begin{array}{l}\text { Discutir as } \\
\text { consequências que a } \\
\text { hospitalização pode } \\
\text { trazer ao paciente } \\
\text { idoso e quais } \\
\text { intervenções podem } \\
\text { prevenir ou } \\
\text { minimizar esses } \\
\text { efeitos indesejados. }\end{array}$ & $\begin{array}{l}\text { Os cuidados hospitalares } \\
\text { hoje são dirigidos } \\
\text { preferencialmente ao } \\
\text { tratamento de doenças } \\
\text { agudas dessa forma } \\
\text { fatores como função } \\
\text { cognitiva e mobilidade, } \\
\text { que mais afetam a } \\
\text { independência e o } \\
\text { prognostico do paciente, } \\
\text { são frequentemente } \\
\text { deixados em segundo } \\
\text { plano. }\end{array}$ \\
\hline 2012 & $\begin{array}{l}\text { LILACS/ } \\
\text { Revista }\end{array}$ & $\begin{array}{c}\text { Menezes, R.L. } \\
\text { D; } \\
\text { Bachion, } \\
\text { M.M. }\end{array}$ & $\begin{array}{l}\text { Ocorrência de } \\
\text { quedas e seu } \\
\text { contexto num } \\
\text { seguimento de dois }\end{array}$ & $\begin{array}{l}\text { Avaliar a ocorrência } \\
\text { de quedas em idosos } \\
\text { institucionalizados } \\
\text { em Goiânia-GO. }\end{array}$ & $\begin{array}{l}\text { Ocorrência de } 54,2 \% \text { de } \\
\text { prevalência de quedas; } \\
\text { predominantemente } \\
\text { durante o dia; Fora do }\end{array}$ \\
\hline
\end{tabular}




\begin{tabular}{|c|c|c|c|c|c|}
\hline & $\begin{array}{l}\text { Eletrônica } \\
\text { de } \\
\text { Enfermagem }\end{array}$ & & $\begin{array}{c}\text { anos em idosos } \\
\text { institucionalizados. }\end{array}$ & & $\begin{array}{c}\text { ambiente interno } \\
\text { institucional; Durante a } \\
\text { deambulação; Em piso de } \\
\text { cimento, seco e a partir de } \\
\text { tropeços quando estavam } \\
\text { calçados de chinelo. }\end{array}$ \\
\hline 2012 & $\begin{array}{c}\text { SciELO / } \\
\text { Revista } \\
\text { Latino } \\
\text { Americana } \\
\text { de } \\
\text { Enfermagem }\end{array}$ & Abreu, C;et al & $\begin{array}{c}\text { Quedas em meio } \\
\text { hospitalar: } \\
\text { Um estudo } \\
\text { longitudinal. }\end{array}$ & $\begin{array}{l}\text { Visou avaliar a } \\
\text { prevalência das } \\
\text { quedas, conhecer as } \\
\text { suas consequências, } \\
\text { identificar as causas } \\
\text { e estudar estratégias } \\
\text { para preveni-las. }\end{array}$ & $\begin{array}{l}\text { Maioria das quedas em } \\
\text { doentes parcialmente } \\
\text { dependentes, entre } 64 \text { e } \\
74 \text { anos. O quarto foi o } \\
\text { local onde } \\
\text { majoritariamente } \\
\text { ocorreram as quedas. }\end{array}$ \\
\hline 2013 & $\begin{array}{l}\text { LILACS/ } \\
\text { Revista } \\
\text { Brasileira de } \\
\text { Ciências da } \\
\text { Saúde }\end{array}$ & $\begin{array}{l}\text { Rodrigues, C.L; } \\
\text { et al }\end{array}$ & $\begin{array}{l}\text { Fatores associados às } \\
\text { quedas em idosos } \\
\text { atendidos em } \\
\text { hospital da Região } \\
\text { Sul da cidade de São } \\
\text { Paulo. }\end{array}$ & $\begin{array}{c}\text { Analise de } \\
\text { prontuários de } \\
\text { pacientes com mais } \\
\text { de } 60 \text { anos, que } \\
\text { tenham sido } \\
\text { atendidos por } \\
\text { motivos de quedas. }\end{array}$ & $\begin{array}{l}\text { Maior frequência de } \\
\text { queda do mesmo nível em } \\
\text { idosos do sexo feminino, } \\
\text { com idade predominante } \\
\text { entre os } 75 \text { anos ou mais. }\end{array}$ \\
\hline 2013 & $\begin{array}{c}\text { SciELO / } \\
\text { Revista } \\
\text { Brasileira de } \\
\text { Enfermagem } \\
\text { REBEN }\end{array}$ & $\begin{array}{c}\text { Chianca, T.C. } \\
\text { M; et al }\end{array}$ & $\begin{array}{c}\text { Prevalência de } \\
\text { quedas em idoso } \\
\text { cadastrados em um } \\
\text { centro de saúde de } \\
\text { Belo } \\
\text { Horizonte - MG. }\end{array}$ & $\begin{array}{l}\text { A queda é utilizada } \\
\text { como indicador de } \\
\text { qualidade e de } \\
\text { qualidade de vida em } \\
\text { serviços } \\
\text { especializados. }\end{array}$ & $\begin{array}{c}\text { Observou-se que } 64 \\
\text { (59,3\%) dos pacientes } \\
\text { idosos já haviam sofrido } \\
\text { queda. }\end{array}$ \\
\hline 2014 & $\begin{array}{l}\text { SciELO/Revis } \\
\text { ta Brasileira } \\
\text { de Ciência e } \\
\text { Saúde } \\
\text { Coletiva. }\end{array}$ & $\begin{array}{c}\text { Gomes, E.C. C; } \\
\text { etal }\end{array}$ & $\begin{array}{l}\text { Fatores associados } \\
\text { ao risco de quedas } \\
\text { em idosos } \\
\text { institucionalizados: } \\
\text { Uma revisão } \\
\text { integrativa. }\end{array}$ & $\begin{array}{l}\text { Identificar os fatores } \\
\text { associados ao risco } \\
\text { de quedas em idosos } \\
\text { institucionalizados. }\end{array}$ & $\begin{array}{l}\text { Fatores relacionados ao } \\
\text { risco de quedas: sexo } \\
\text { feminino; diagnóstico de } \\
\text { doença crônica; uso de } \\
\text { benzodiazepínicos; queda } \\
\text { anterior; restrições de } \\
\text { mobilidade. }\end{array}$ \\
\hline
\end{tabular}

A discussão dos resultados será categorizada em subtópicos, de acordo com a evolução teórica apresentada pela amostra, de modo a permitir uma construção ideológica clara a respeito dos fatores de risco para queda em idosos hospitalizados.

\subsection{Envelhecimento e fatores de risco para a queda}

Enquanto a expectativa de vida da população idosa tem aumentado em função das intervenções médicas e de saúde publica, estudo sobre os fatores de risco para quedas em idosos hospitalizados tem surgido como meio de oportunizar a melhoria da qualidade de vida neste grupo populacional. Entre os fatores associados às quedas, estão: polifarmácia, múltiplas patologias, comprometimento cognitivo, diminuição da acuidade visual, fraqueza óssea, dificuldade para deambulação e barreiras ambientais, como, por exemplo, o uso de tapetes domésticos. A maioria das quedas ocorre durante as atividades rotineiras dos idosos e gera o medo de cair novamente ${ }^{12}$.

Conforme a idade avança, aumenta a incidência de quedas. Tal relação explica-se pelos efeitos cumulativos das alterações relacionadas à idade, as doenças e ao meio ambiente inadequado. Com envelhecimento, ocorrem perdas de células musculares e elasticidade dos tecidos diminuição da massa óssea alterações posturais e redução da mobilidade articular, sistemas esses responsáveis pela mobilidade do corpo. Portanto, a degradação desses 
componentes parece predispor o idoso a uma maior incidência de queda. A fraqueza muscular dos idosos hospitalizados estabelece uma relação bidirecional com suas perdas funcionais, dependência de cuidadores, sedentarismo e aceleração do envelhecimento fisiológico ${ }^{13-14}$.

\subsection{Fator sexo e idade}

Com base nos artigos selecionados, três relatam que há uma predominância de queda em idosos do sexo feminino, considerado este gênero como frágil, pois está relacionado à maior prevalência de osteoartrose, diminuição de força e de massa muscular, seu maior vínculo com as atividades domésticas, alterações hormonais como a redução do estrógeno com consequente perda da massa óssea. Além disso, a população de mulheres, no Brasil, é maior do que a de homens e sua esperança de vida é elevada, o que ajuda a entender porque, da população feminina sofrer mais com as alterações do envelhecimento, ${ }^{8,15-16}$.

Alguns autores apontam que a idade superior a 65 anos e a pluralidade de patologias são importantes fatores de risco às quedas. Presença de doenças crônico-degenerativas (hipertensão, diabetes, artrite e demência), além do uso concomitante de vários medicamentos (polifarmácia) podem acarretar uma maior vulnerabilidade para queda, além do mais, no meio hospitalar, o idoso enfrenta um ambiente estranho e muitas vezes a falta de família, tornando-o mais vulnerável à queda ${ }^{18-19}$.

\subsection{Turno de ocorrência, superfície de contato e instabilidade}

Em relação ao turno em que ocorreram as quedas, houve uma predominância no turno da noite. Autores revelam que durante a noite, o número de profissionais geralmente é reduzido e com isso, a vigilância e visitas aos leitos diminuem, resultando em um aumento da suscetibilidade para queda. O local desconhecido e o fato do turno noturno ser visto como um período de silêncio e escuridão, o paciente se sente constrangido em solicitar auxilio dos profissionais, resultando em aumento das chances de cair ${ }^{6,18}$.

De acordo com os artigos estudados, o piso escorregadio, irregular e "liso" das instituições hospitalares é reconhecido pela literatura como fator predisponente a quedas. O cimento e o asfalto são as superfícies empregadas majoritariamente nos ambiente externos, ocasionando uma diferença tátil no equilíbrio do idoso em relação ao seu ambiente de origem. O calçado também contribui para instabilidade postural e queda, todavia, é pouco discutido na literatura, apesar de uma grande proporção de quedas ocorrerem durante a caminhada ou quando se transpõem obstáculos comuns, tais como calçadas e/ou escadas ${ }^{20}$.

\subsection{Papel profissional/institucional}

A assistência ao idoso deve ter como objetivo a manutenção e valorização da autonomia. Para tanto, é necessário avaliar o grau de dependência e instituir medidas voltadas para o alcance do maior grau possível de independência funcional e autonomia. Dessa forma, para que os trabalhadores de saúde possam contemplar os novos paradigmas da atenção à saúde da pessoa idosa e contribuir para a promoção do envelhecimento saudável é importante que eles conheçam, inicialmente, a maneira pela qual a mudança do perfil demográfico tem influenciado a sua área de abrangência, investigando o aumento progressivo de idosos e no desenvolvimento de um plano de 
intervenção que tente priorizar a melhoria da qualidade de vida, bem como o envelhecimento ativo, mantendo a capacidade funcional do idoso ${ }^{21}$.

A ocorrência de queda é um evento relativamente frequente nas instituições hospitalares, e chega a ter consequências expressivas nos componentes físicos, psicológicos e pessoais. Para tanto, os serviços de saúde deverão estar preparados para identificá-los e promover-lhes assistência diferenciada e pautada na participação de diferentes profissionais da área da saúde 22 . De acordo com o protocolo de prevenção de queda da Agência Nacional de Vigilância Sanitária (ANVISA), as unidades de saúde, orientadas pelo seu Núcleo de Segurança do Paciente, deverão adotar medidas gerais para prevenção de quedas de todos os pacientes independentes do risco ${ }^{23}$.

\section{CONSIDERAÇÕES FINAIS}

A queda é uma das principais causas de mortes da população idosa. De origem multifatorial e complexa, sua prevenção é por vezes dificultada. No entanto, entende-se que tais eventos ainda são negligenciados.

Neste sentido, esta pesquisa identificou, diante dos trabalhos coletados, que apesar dos fatores de riscos serem reconhecidos, há ainda lacunas em relação aos estudos sobre quedas em idosos hospitalizados, pois apenas nove dos trinta trabalhos trazem essa discussão, o que comprova, não apenas a necessidade de mais estudos nessa temática, como uma maior ampliação das ações desenvolvidas para saúde dos idosos. De todo modo, pode-se destacar como fatores de risco para queda nos idosos hospitalizados: polifarmácia, múltiplas patologias, comprometimento cognitivo, diminuição da acuidade visual, fraqueza óssea, dificuldade para deambulação e barreiras ambientais, ser do sexo feminino, com idade superior a 65 anos, atividades no turno noturno e alterações de superfícies de deambulação.

Deste modo, conclui-se que as quedas em idosos hospitalizados acorrem, muitas vezes, devido à falta de medidas preventivas que podem ser implementadas pelas instituições, assim como, os órgãos competentes devem intensificar os programas voltados para a população idosa, uma vez que seu crescimento é notório e consequentemente suas necessidades no que se refere à manutenção da qualidade de vida.

\section{REFERÊNCIAS BIBLIOGRÁFICAS}

1. BRASIL, MINISTÉRIO DA SAÚDE. Caderneta de Saúde da Pessoa Idosa. 1 ed. Governo Federal, Brasília-DF, 2010. Disponível em: <http://bvsms.saude.gov.br/bvs/publicacoes/ caderneta_saude_pessoa_idosa.pdf>. Acesso em: 10 de Dezembro de 2014.

2. IBGE-Instituto Brasileiro de Geografia e Estatística - Síntese de Indicadores Sociais, uma Análise das Condições de Vida da População Brasileira. Governo Federal/Brasília-DF, 2013. Disponível em: <ftp://ftp.ibge.gov.br/Indicadores_Sociais/Sintese_de_Indicadores_Sociais_2013/ SIS_2013.pdf>. Acesso em: 06 de Dezembro de 2014.

3. BATISTA, N.C., CRISPIM, N.F. As Interferências das Relações Familiares no Processo do Envelhecer. Revista Kairos Gerontologia, v.15, n.5, p.169-189, Jun.2012.

4. CAMPOLINA, A.G., ADAMI, F., SANTOS, J.L.F., LEBRÃO, M.L.A. Transição de saúde e as mudanças na expectativa de vida saudável da população idosa: Possíveis impactos da 
prevenção de Doença crônicas. Cadernos de Saúde Pública, v.29, n.6, p.1217-1229, Jun.2013.

5. PROCHET, C.T., SILVA, M.J.P., FERREIRA, D.M., EVANGELISTA, V.C. Afetividade no processo de cuidado do idoso na compreensão da enfermeira. Revista Escola Enfermagem-USP, v.46, n.1, p.96-102, 2012.

6. ABREU, C., MENDES, A., MONTEIRO, J., SANTOS, F.R. Queda em meio hospitalar: um estudo longitudinal. Revista Latino-Americana de Enfermagem, v.20, n.3, p.1-7, Maio-Jun.2012.

7. ALMEIDA, S.T., SOLDERA, C.L.C., CARLI, G.A., GOMES, I., RESENDE, T.L. Análise de fatores extrínsecos e intrínsecos que predispõe a queda em idosos, Rev. Assoc. Med. Bras. v.58, n.4, p.427-433, Jul-Ago.2012.

8. ALMEIDA, R.A.R., ABREU, C.C.F., MENDES, A.M.O.C. Queda em doentes hospitalizados: contributos para prática baseada na prevenção. Revista de Enfermagem Referência, v.2, n.3, p. 2163-2172, 2010.

9. GOMES, E.C.C., MARQUES, A.P.O., LEAL, M.C.C., BARROS, B.P. Fatores associados ao risco de quedas em idosos institucionalizados: Uma revisão integrativa. Ciência \& Saúde Coletiva, v.19, n.8, p.3543-3551, 2014.

10. MENDES, K.D.S., SILVEIRA, R.C.C.P., GALVÃO, C.M. Revisão integrativa: métodos de pesquisa para incorporação de evidências na saúde e na enfermagem. Texto \& contexto Enfermagem, v.17, n.4, p.758-64, 2008.

11. SOUZA, M.T., SILVA, M.D., CARVALHO, R. Revisão integrativa: O que é e como fazer? Einstein, v.80, n.1, p.102-6, 2010.

12. MALLMANN D.G., HAMMERCHMIDT, K.S.A., SANTOS, S.S.C. Instrumento de avaliação de quedas para idosos (IAQI): enfermeiro analisando vulnerabilidade e fragilidade. Rev. Bras. Geriatria e Gerontologia, v.15, n.3, p.517-527, 2012.

13. RODRIGUES, C.L., FERNANDES, A., ARMOND, J.E., GORIOS, C. Fatores associados a quedas em idosos atendidos em um hospital da região do sul da cidade de São Paulo. Revista Brasileira de Ciências da Saúde, v.11, n.36, p.14-19, Abr-Jun, 2013.

14. FERREIRA, L.L., SANCHES, A.G., MARCINDES, L.P., SAAD, P.C.B. Risco de queda em idosos com doença de Alzheimer institucionalizados. Revista Fisioterapia \& Saúde Funcional, v.12, n.3, p.379-385, 2013.

15. ALMEIDA, L.P., BRITES, M.F., TAKAZAWA, M.G.M.H. Quedas em idosos: fatores de risco, RBCEH. v.8, n.3, p.384-391, Set-Dez. 2011.

16. LUZIA, M.F., VICTOR, M.A.G., LUCENA, A.F. Diagnóstico de enfermagem risco de quedas: prevalência e perfil clínico de paciente hospitalizados. Revista Latino-Americana Enfermagem, v.22, n.2, p.262-8, Mar-Abr. 2014.

17. QUEIROZ, L., LIRA, S., SASAKI, A. Identificação do risco de quedas pela avaliação da mobilidade funcional em idosos hospitalizados. Revista Baiana de Saúde Pública, v.33, n.4 p.534-543, OutDez, 2010.

18. COSTA, S.G.R.F., MONTEIRO, D.R., HEMESATH, M.P., ALMEIDA, M.A. Caracterização das quedas do leito sofrida por pacientes internados em um hospital universitário. Revista Gaúcha de Enfermagem, v.32, n.4, p.676-81, Dez, 2011.

19. JUNIOR-CÉSAR, A.B., FOLCHINI, A.B., RUEDIGER, R. R. Estudos comparativos entre o trauma 
em idosos e não idosos atendidos em um Hospital Universitário de Curitiba. Rev. Col. Bras. Cir., v.40, n.4, p.281-286, 2013.

20. MENEZES, R.L., BACHION, M.M., Ocorrências de quedas e seu contexto num seguimento de dois anos em idosos institucionalizados. Revista Eletrônica de Enfermagem, v.14, n.3, p.550-8, Jul-Set.2012.

21. RINALDI, F. C., CAMPOS, M. E. C., LIMA, S.S., SODRÉ, F.S.S. O papel da enfermagem e sua contribuição para a promoção do envelhecimento saudável e ativo. Revista Eletrônica Gestão \& Saúde, V.4, n.2, p.454-66.2013.

22. STHAL, H.C., BERTI, H.W., PALLHARES, V.C. Grau de dependência de idosos hospitalizados para realização das atividades básicas da vida diária. Texto \& Contexto Enfermagem, v.20, n.1, p.5967, Jan-Mar.2011.

23. Brasil. Ministério da Saúde. Documento de referência para o Programa Nacional de Segurança do Paciente. 1. ed. Fundação Oswaldo Cruz; Agência Nacional de Vigilância Sanitária. - BrasíliaDF, 2014. 40p. Disponível em: <http://www.rets.org.br/sites/default/files/ PPT_COLETIVA_SEGURANCA_PACIENTE_FINAL.pdf>. Acesso em: 09 de Dezembro de 2014.

\section{Referências da amostra não inclusas no texto}

24. SALES, M.V.C., SILVA, T.J.A., GIL-JÚNIOR, L.A., JACOB-FILHO, W. Efeitos adversos da internação hospitalar para o idoso. Geriatria \& Gerontologia, v.4, n.4, p.238-46. 2010

25. CHIANCA, T.C.M., ANDRADE, C.R., ALBUQUERQUE, J., WENCESLAU, L.C.C., TADEU, L.F.R., MACIEIRA, T.G.R., ERCOLE, F.F. Prevalência de quedas em idosos cadastrados em um Centro de Saúde de Belo Horizonte-MG. Revista Brasileira de Enfermagem, v.66, n.2, p.234-40. MarAbr. 2013. 